(Aus der chirurgischen Klinik zu Halle a. \$. [Direktor: Professor Dr. Voelcker].)

\title{
Über die angeborenen Contracturen des Hüft- und Kniegelenks.
}

\author{
Von \\ Privatdozent Dr. Friedrich Loeffler.
}

Mit 2 Textabbildungen.

Unter den angeborenen Deformitäten gehören die angeborenen Contracturen der Gelenke, besonders des Hüft- und Kniegelenks zu den großen Seltenheiten. Lassen wir nun noch. die Fälle unberücksichtigt, bei denen derartige Gelenkcontracturen nur ein Glied in der Kette mehrerer Deformitäten an ein und demselben Individuum sind, wo sie das Krankheitsbild keineswegs beherrschen, so würde die Zahl der Fälle von angeborenen Gelenkcontracturen sich erheblich vermindern. Beobachtungen von multiplen Contracturen in fast allen größeren Gelenken, die dann noch durch allerlei Bildungsfehler, wie Muskel- und Knochendefekte an den Extremitäten, durch Aplasien und Hemmungsbildungen, besonders auch an Kopf und Rumpf (deformer Schädel, Spina bifida, Atresia ani u. a.) kompliziert sind, sind von Redard, Lu merzheim, Schanz, Phocas, Paucot, Wunsch, Magnus mitgeteilt worden. Sie haben immerhin für die Frage der Ätiologie insofern Bedeutung, als sie uns die Entstehung derselben durch fehlerhafte Keimanlage überzeugend begründen. Viel seltener und ätiologisch noch wenig geklärt sind die reinen, durch Schrumpfungsprozesse im Muskel mit sekundärer Verkürzung verursachten Gelenkcontracturen. Rosenfeld fand unter 4400 Deformitäten keinen derartigen Fall. König schreibt sogar den angeborenen Kniecontracturen mehr das Interesse einer Kuriosität zu. Auch Gaugele hält die angeborenen Gelenkcontracturen für äußerst selten.

Allgemein nimmt man an, daß die reinen muskulären Gelenkcontracturen durch eine Zwangslage des Kindes im Uterus bei Verminderung des Fruchtwassers verursacht sind. Für die Streckcontracturen, die am häufigsten beobachtet sind, ist es gelungen, die typische Zwangshaltung des Kindes, die es im Uterus gehabt haben muß, nach der Geburt herzustellen. Anders aber verhält es sich schon bei den angeborenen Beugecontracturen im Hüft- und Kniegelenk. Die hierüber beobachteten Fälle sind noch zu gering, um die Entstehung dieser Contracturen durch eine typische Zwangslage erklären zu können. 
Nur durch weitere Mitteilungen reiner angeborener Gelenkcontracturen, die durch keine, auf fehlerhafte Keimanlage hinweisende Mißbildungen kompliziert sind, besonders auch durch genaue Angaben über Schwangerschaft, Geburtsverlauf und Haltung des Kindes nach der Geburt wird es gelingen, die Entstehungsweise dieser Contracturen zu erklären.

Ich will daher versuchen, an Hand zweier von mir beobachteten Fälle von reinen angeborenen Contracturen des Kniegelenks in Streckund Beugestellung zur Aufklärung über die Entstehung dieser seltenen Deformitäten beizutragen.

1. Fall: Angeborene Streckcontractur beider Kniegelenke, leichte Deformität beider Füße.

Vorgeschich te : Die Familienanamnese ist ohne Besonderheiten. Deformitäten sind nicht in der Familie vorgekommen. Es handeit sich ium das dritte, 14 Tage alte Kind (Knabe) gesunder Eltern. Die Schwangerschaft ist normal verlaufen. Die Fruchtwassermenge bei der Geburt, die gänz normal rerlief und obne Kunsthilfe erfolgte, zeigte keinen auffälligen Mangel. Die Geburt erfolgte in Steißlage. Gleich nach der Geburt fiel der Mutter an dem Kinde die seltsame Haltung der Beine auf. Beide Beine waren stark im Hüftgelenk gebeugt, ïber Bauch und Brust

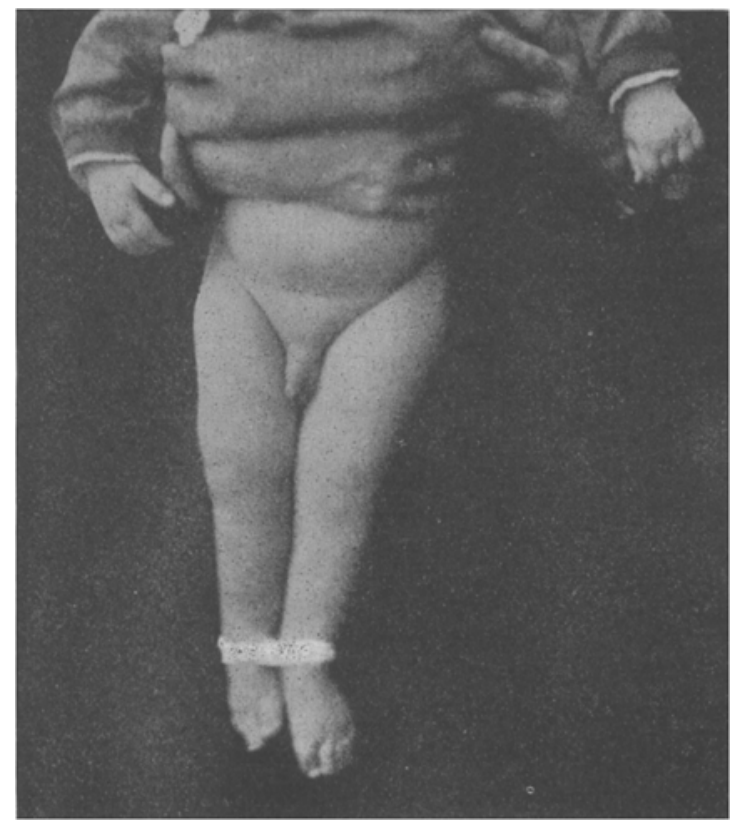

Abb. 1. vielleicht sogar etwas überstreckt. Das rechte Kniegelenk stand in leichter X-Stellung. ..Eine Beugung der Kniegelenke war aktiv und passiv ganz unmöglich, dagegen ließ sich die U̇berstreckung noch um einige der linken Schulter lagen. Passiv konnten die Beine in eine normale gestreckte Lage gebracht werden. Diese Lage nahm das Kind näch einigen Tagen auch aktiv ein. Dagegen blieb aber noch eine eigentümliche Stellung. der Kniegelenke und Füße übrig; die Kniegelenke konnten auch passiv nicht gebeugt werden. Aus diesem Grunde kam die Mutter in die Klinik. Aufnahmebefund: (siehe Abb. 1).

Kräftiges, gut entwickeltes Kind. Mißbildungen am Knochensystem oder an den Weichteilen ließen sich nicht nachweisen. Beide Beine waren im Kniegelenk vollständig geso daß die Füße auf 
Grade vornehmen. Beim Abtasten der Gelenke war eine abnorme Stellung der Ober- und Unterschenkelgelenkflächen nicht festzustellen. Die Oberschenkelgelenkkondylen waren deutlich hindurchzufühlen, doch konnte von einer Subluxation, geschweige denn Luxation im Kniegelenk nicht gesprochen werden. Das Röntgenbild der Kniegelenke in zwei senkrecht aufeinanderstehenden Ebenen ließ die Epiphysenkerne klar erkennen, ihre Lage zueinander war vollständig normal, d. h. sie standen sich bei der Betrachtung von vorn und von der Seite genau gegenüber. Also bestand keine Subluxation oder Luxation im Kniegelenk. Die Hüftgelenke waren passiv und aktiv normal beweglich. Der rechte Fuß stand in Supinationsstellung, der linke in Pronationsstellung. Beide. Füße ließen sich leicht adaptieren, und man konnte erkennen, wie ihre Lage im Uterus gewesen war. Auffällig war vielleicht noch bei seitlicher Betrachtung der Beine, daß die Quadricepsmuskulatur im oberen Teil dicker und wulstartiger war als am unteren Teil.

Um gleich die Behandlung zu besprechen, so habe ich die Füße redressiert und den rechten Fu $B$ in starker Pronations- und Dorsalflexionsstellung eingegipst, den linken in starker Supinations- und Plantarflexionsstellung. Nach achtwöchentlicher Gipsbehandlung standen die Füße in guter, leicht überkorrigier. ter Stellung. Die Kniestreckcontracturen habe ich manuell etwas zu beugen versucht und dann der

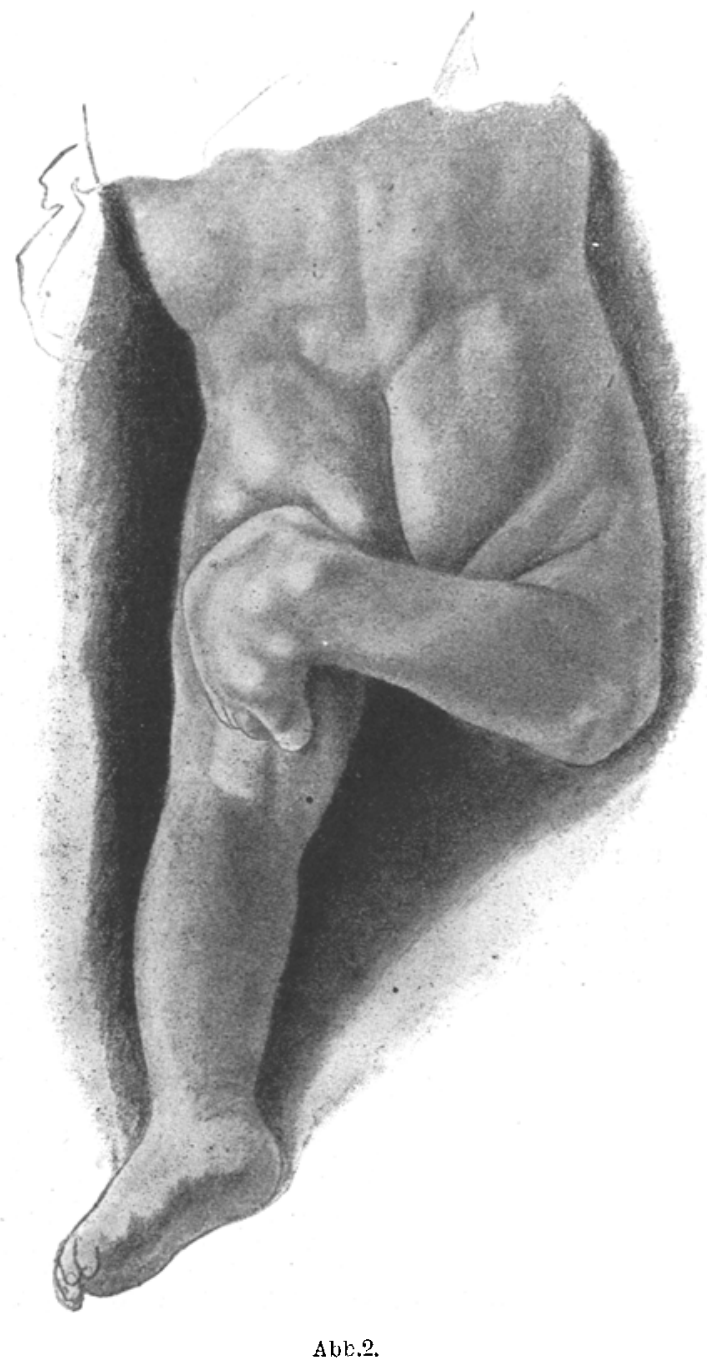
Mutter geraten, Massage und Beugeübungen täglich zu Hause fortzusetzen. Jetzt, nach 3 Monaten, kann das Kind die Knie aktiv und passiv bis etwa $95^{\circ}$ beugen.

2. Fall: Angeborene Beugecontractur im rechten Kniegelenk, Supinationsstellung des rechten Fußes.

Vorgeschichte: Die Familienanamnese erwies sich ohne Besonderheiten, in der Familie sind keine Mißbildungen vorgelzommen. Zwei Geschwister leben 
und sind gesund. Die Schwangerschaft der Mutter und die Geburt des Kindes verliefen normal. Über Fruchtwassermenge können keine Angaben mehr gemacht werden. Da seit der Geburt eine abnorme Stellung des rechten Unterschenkels besteht, wurde das Kind in die Klinik zur Begutachtung gebracht.

Bef und: (siehe Abb. 2). 8 Tage alter, gesunder, kräftiger Knabe. Die Untersuchung des Knochensystems ergab einen normalen Befund. Es bestanden keine anderen Mißbildungen. Das rechte Kniegelenk stand in einer Beugestellung von $70^{\circ}$. Der Unterschenkel war einwärts rotiert, so daß der rechte Fuß, der in starker Supinationsstellung stand, auf die linke Gesäßmuskulatur zu liegen kam. Durch den Druck des Fußes war die linke Gesäßhälfte schwächer entwickelt als rechts. Nach Fortnehmen des Fußes sah man hier eine dentliche Delle. Ließ man den FuB los, so federte er sofort wieder zurück. Aktiv konnte das Kniegelenk nur wenig mehr gestreckt werden, auch passiv war eine Streckung nur bis $80^{\circ}$ möglich. Dann fühlte man deutlich, wie die medialen Unterschenkelbeuger sich stark anspannten und als straffer, fester Sehnenstrang hervorsprangen. Ein Anspannen der lateralen Beuger war nicht so stark zu fühlen.

Eine weitere Beugung des Kniegelenks war passiv noch möglich. Bei der Abtastung des Kniegelenks ließ sich eine abnorme Verschiebung der Gelenkflächen nicht feststellen. Der Beugestellung entsprechend ließen sich die Oberschenkelgelenkkondylen besonders gut abtasten. Auch auf den Röntgenbildern war eine Subluxation oder gar Luxation durch falsche Stellung der Epiphysenkerne nicht zu sehen. Die Bewegung im rechten Hüftgelenk war normal.

Behandlung: In Athernarkose nabm ich eine offene Tenotomie der medialen Unterschenkelbeuger mit Resektion eines $2 \mathrm{~cm}$ langen Sehnen- und Muskelstückes vor. Das Kniegelenk läßt sich unter leichter manueller Redression fast vollständig strecken. Nach der Hautnaht erfolgte die Redression des in Supinationsstellung stehenden rechten Fußes. Ein Gipsverband wurde in fast normaler gestreckter Kniestellung und leichter Pronations-Dorsalflexionsstellung des rechten Fußes angelegt.

Die Fälle von angeborener Contractur des Kniegelenks in Streckstellung finden wir teils unter dem Titel , angeborene Luxationen des Kniegelenks", teils unter ,kongenitale Contracturen" veröfentlicht. Es herrscht also schon in der Nomenklatur einige Verwirrung. Nasse behauptet schon, daß die meisten Fälle, die als zur ersten Gruppe gehörig beschrieben worden sind, keine wahren Luxationen in dem Sinne waren, daß ein wirkliches Abweiehen der Gelenkflächen voneinander stattgefunden hätte. Sie sind vielmehr als Contracturen aufzufassen, bei denen allerdings geringe Grade der Verschiebung, Subluxationen, vielfach vorhanden waren. Ebenso sind bei den als „kongenitale Contracturen" beschriebenen Fällen häufig Subluxationen beobachtet worden. Die Contracturen des Kniegelenks in Hyperextension zeigen im allgemeinen dasselbe klinische Bild wie die sog. präfemorale Luxation der Tibia, bei der stets eine mehr oder weniger starke Hyperextension besteht. Muskat schlägt daher vor, derartige Fälle unter ,Genu recurvatum congenitum" zusammenzufassen.

Der Grund für die Unsicherheit, zu welcher Gruppe man die angeborenen Contracturen des Kniegelenks in Streckstellung rechnen soll, scheint mir darin zu liegen, daß die Beobachtung dieser Fälle in eine 
Zeit fällt, in der die Röntgenstrahlen noch nicht entdeckt waren, oder es noch nicht möglich war, alle die Feinheiten am Skelettsystem zu erkennen, wie wir es heute durch die Entwicklung der Röntgentechnik imstande sind. Man war nur auf den klinischen Befund angewiesen, und dieser zeigte wohl meistens das Bild eines im Kniegelenk hyperextendierten Beines. Durch Palpation eines solchen Kniegelenks hat man wohl festgestellt, daß die Oberschenkelkondylen bei dem einen Fall mehr, bei dem anderen weniger stark nach hinten vorsprangen, und diesen Befund als angeborene Luxation oder angeborene Contractur angesehen.

Heutzutage sind wir imstande, durch das Röntgenbild eines Kniegelenks in zwei zueinander senkrecht stehenden Ebenen, von vorn und seitlich, uns über die Stellung der Gelenkflächen zueinander sicher zu überzeugen.

Wir wissen, daß der distale Epiphysenkern des Oberschenkels sich zuerst entwickelt. Später, aber auch noch vor der Geburt, entwickelt sich der proximale Epiphysenkern der Tibiagelenkfläche. Wir können also diese kleinen Epiphysenkerne bei einem Neugeborenen röntgenologisch darstellen und somit erkennen, ob eine Luxation vorhanden ist oder nicht. Durch die Fortschritte der Röntgentechnik sind wir also in der Lage zu unterscheiden, ob eine angeborene Contractur oder eine angeborene Luxation vorliegt.

Wie kommt aber die Streckcontractur zustande? Aus der Lage des Kindes gleich nach der Geburt wissen wir, daß im Uterus die Beine im Hüftgelenk hochgradig flektiert über Bauch und Brust nach oben geschlagen gelegen haben. So wird also zunächst $d$ urch diese Zwangs lage eine Hyperextension im Kniegelenk hervorgerufen, die bei verstärktem Druck infolge Fruchtwassermangels oder bei längerer Dauer schließlich zur Luxation oder Subluxation führt.

Finden wir nach der Geburt eine Luxation im Kniegelenk und Behinderung der Kniebeugung vor, so können wir uns diese durch die Luxationsstellung erklären. Auch eine Subluxation mit starker Hyperextension des Kniegelenks kann dadurch zur Streckcontractur führen, daß die Zugrichtung der Unterschenkelbeuger verändert wird, und diese nicht mehr hinter den Oberschenkelkondylen, wie normalerweise, sondern vor diesen verlaufen und somit als Überstrecker wirken. Barth hat bei der pathologisch-anatomischen Untersuchung eines Genu recurvatum congenitum gefunden, daß die Epiphyse des Femur an die Diaphyse in einem nach vorn offenen Winkel angesetzt war. Durch diese Verbiegung war die Gelenkfläche der Tibia ebenfalls nach vorn gekehrt.

Liegt also eine Luxation oder Subluxation im Kniegelenk, vielleicht noch mit Muskelverlagerung oder eine Gelenkumbildung vor, so können 
wir uns das Zustandekommen einer Kniestreckcontractur erklären. Ich will dabei unberücksichtigt lassen, ob nicht diese Befunde als sekundäre Erscheinungen aufzufassen sind und zwar als fortgeschrittenere Stadien einer anfänglich einfachen Kniestreckcontractur infolge länger dauernden Druckes bei der Zwangslage des Kindes.

Sind aber diese Befunde nicht vorhanden, so müssen wir die angeborenen Streckcontracturen des Kniegelenks als rein myogenen Ursprungs annehmen, sei es, daß durch intrauterine Zwangslage, sei es, daß durch abnorme Druckwirkung oder durch beides zusammen eine Schrumpfung des Muskels stattgefunden hat. Dann bleibt aber noch die Beantwortung der Frage offen: Warum finden wir nicht bei derartigen Fällen auch Contracturen des Hüftgelenks, und warum finden wir bei Klumpfußkindern, die doch auch einem starken intrauterinen Druck ausgesetzt waren, fast niemals Beugecontracturen der Hüft- und Kniegelenke?

Mit noch mehr Berechtigung können wir uns diese Frage vorlegen, und noch schwieriger wird ihre Beantwortung sein, wenn wir erfahren, daß die Kniebeugecontracturen so überaus selten sind. Eine den angeborenen Streckcontracturen des Kniegelenks analoge Erklärung durch gewaltsame Beugung des Kniegelenks mit sekundärer Luxation oder Subluxation mit Muskelverschiebung läßt der Bewegungsmodus des Kniegelenks schwer zu. So sind wir denn bei der Erklärung des Zustandekommens einer angeborenen Kniebeugecontractur noch sehr im unklaren, zumal die davon beobachteten Fälle recht vereinzelt sind. Ich sehe dabei wieder von den komplizierten Krankheitsfällen $a b$, bei denen die Kniebeugecontractur in Gemeinschaft mit zahlreichen anderen angeborenen Mißbildungen auftritt und demnach auf eine fehlerhafte Keimanlage oder schwere Entwicklungsstörung zurückzuführen ist.

Auch bei meinem Fall kann infolge Fehlens jeglicher anderen Mißbildung des Knochensystems von der Annahme eines primären Bildungsfehlers Abstand genommen werden.

Die Begründung angeborener Contracturen der Kniegelenksbeugemuskeln durch Entwicklungsstörungen des fötalen Nervensystems, wie es Potel versucht hat, ist bei meinem Fall von der Hand zu weisen, denn es lagen keine Störungen der normalen Nervenfunktion vor.

Desgleichen ließen sich keine Anhaltspunkte für eine fötale Knochenerkrankung finden.

Es wird daher auch für das Zustandekommen einer angeborenen Kniebeugecontractur die bei weitem häufigste Ursache, und zwar eine intra-uterine Zwangslage, angenommen. Diese kann, sowohl durch die falsche Lage des Kindes im Uterus, als auch durch Behinderung der freien Bewegung infolge räumlicher Beschränkung zur Contractur führen.

Auf Grund der beiden von mir beobachteten Fälle glaube ich die Richtigkeit dieser Annahme bestätigen zu können. Ich muß aber hinzu- 
fügen, daß es nur eine ganz bestimmte $Z_{\text {wangslage sein kann, die }}$ jedesmal zu einer Contractur des betreffenden Muskels führt. Wir dürfen zur Erklärung der angeborenen Gelenkcontracturen nun nicht die Fälle heranziehen, bei denen sich gleichzeitig Contracturen in mehreren Gelenken finden (Hüft- und Kniegelenk), da meiner Meinung nach hier noch ein anderer, bis jetzt nicht berücksichtigter Faktor in Frage kommt, sondern wir müssen die Fälle betrachten, die nur eine Contractur, im Hüft- oder im Kniegelenk zeigen. Ich glaube für derartige angeborene Contracturen ganz typische $Z_{\text {wangslagen anführen }}$ zu können, für deren Richtigkeit ich zum Teil meine beiden Fälle, zum Teil die eindeutigen aus der Literatur zum Beweis heranziehen kann.

Bei den angeborenen Streckcontracturen im Kniegelenk finden wir stets die Beobachtung mitgeteilt, daß die Beine mit gestreckten Kniegelenken in beiden Hüftgelenken stark flektiert und über Brust und Bauch nach oben geschlagen waren, wie ich es auch bei meinem Fall beobachten konnte. Da diese Zwangslage von allen Zwangslagen des Kindes mit am häufigsten ist, so sind denn auch die dabei sich findenden Streckcontracturen im Kniegelenk am häufigsten beobachtet und durch diese Zwangslage verständlich erklärt worden.

Bei den Beugecontracturen im Hüftgelenk findet man als typische Zwangslage die im Hüftgelenk stark flektierte, abd uzierte und nach a $\mathbf{u}$ Ben rotierte Lage der Beine angegeben.

Die Beobachtungen von isolierten Kniebe ugecontracturen sind leider sehr spärlich. Daher läßt sich für das Zustandekommen dieser angeborenen Contracturen auch noch keine Zwangslage zu einer einheitlichen Erklärung anführen. Durch die Beobachtung an meinem Fall glaube ich aber auch hierfür die dabei gefundene Zwangslage als typisch ansehen zu können, nämlich Streckung, vielleicht leichte Überstreckung im Hüftgelen k und starke Be ug ung im Kniegelen k. Daß diese Zwangslage sicherlich schon lange vor der Geburt bestanden hat, schließe ich aus der durch den Druck des Fußes hervorgerufenen Atrophie der linken Gesäßmuskulatur.

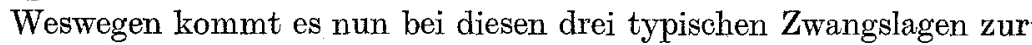
Bildung einer Contractur in den betreffenden Gelenken ? Hierfür gibt uns die Entwicklungsmechanik die Erklärung.

Aus der Entwicklungsmechanik wissen wir, daß durch jede oft wiederholte Bewegung eines Muskels eine Erhöhung seiner Ernährung veranlaßt wird durch vermehrten Zufluß von Nahrungsstoffen, wodurch der Muskel dann wächst. Jede Behinderung der Muskelbewegung setzt also die Zufuhr von Nahrungsstoffen herab, und diese wiederum ruft ein Zurückbleiben des Muskels in seinem Wachstum hervor. So bleibt also der Muskel in seiner Ernährung und damit in seinem Wachstum zurück, der durch die Zwangslage in seiner Funktion gestört wird. 
Nun nimmt ein Kind schon bei normaler Lage im Uterus eine Art Zwangslage ein. Es hält nämlich die Beine im Hüft- und Kniegelenk stark flektiert und die Unterschenkel gekreuzt. Selbst bei kräftigem intra-uterinen Druck, wie er bei Klumpfußkindern bestanden haben muß, kommt es aber deshalb nicht zu Contracturen im Hüft- und Kniegelenk, weil die einzelnen Muskelgruppen der unteren Extremität sich in einer "Mittelstellung " befinden, d. h. durch die Lage wird eine Funktion der Muskeln ausgeschaltet, dagegen bleibt ihnen noch eine zweite Funktion erhalten. Z. B. durch die Beugung im Hüftgelenk wird der Quadricepsmuskulatur die Funktion der Oberschenkelbeugung unterbunden, dagegen wird ihr durch die starke Beugung im Kniegelenk die zweite Funktion, die Unterschenkelstreckung noch erhalten. Ebenso wird der Unterschenkelbeugemuskulatur durch die starke Beugung im Kniegelenk die Funktion der Beugung genommen, und es bleibt ihr noch die Funktion der Oberschenkelstreckung. Es finden sich also somit bei der gewöhnlichen Lage des Kindeș im Uterus die Muskeln in einer sog. „,neutralen Stellung “.

Soll es also zu einer Contracturstellung in dem betreffenden Gelenk kommen, so muß dem Muskel seine zweite Funktion durch die Zwangslage auch noch genommen werden. Es tritt dann eine anormale Zwangslage ein, durch die der Ursprungs- und Ansatzpunkt des betreffënden Muskels extrem genähert sind.

Denn die Entwicklung eines Muskels wird durch seine direkte Anpassung an die gegebene Beweglichkeit seiner Anheftungspunkte bestimmt. Diese Anpassung hängt von der Verringerung der Entfernung von Ursprungs- und Insertionspunkt ab. Sie kann zustande kommen

1. durch einfaches Zurückbleiben im Wachstum,

2. unter Beteiligung von Schrumpfung,

3. auch noch unter Mitwirkung von sehniger Atrophie des Muskels.

Bei der für die Entstehung einer Streckcontractur im Kniegelenk oben angeführten typischen Zwangslage finden wir den Ursprungs- und Ansatzpunkt des diese Contractur verursachenden Muskels aufs äußerste genähert und den Muskel seiner beiden Funktionen beraubt. Denn der Quadriceps, besonders der Rectus femoris, besitzt die Fähigkeit, den Oberschenkel zu beugen und den Unterschenkel zu strecken. Sind die Beine nun im Hüftgelenk stark flektiert und im Kniegelenk überstreckt, über Bauch und Brust nach oben geschlagen, so ist die Entfernung von Ursprung und Ansatz des Rectus femoris auf das äußerste genähert.

Bei den Beugecontracturen im Hüftgelenk wird der Ursprung und Ansatz des Iliopsoas dann am meisten genähert, wenn das Bein im Hüftgelenk stark flektiert und außen rotiert liegt. 
Durch die Streckung des Oberschenkels und die starke Beugung des Unterschenkels bei einer typischen Zwangslage der Kniebeugecontractur werden die Beugemuskeln am stärksten verkürzt und sich somit dieser äußersten Verringerung der Entfernung ihrer Ursprungspunkte funktionell anpassen.

Natürlich kann jede dieser typischen anormalen Zwangslagen durch Fruchtwassermangel verstärkt werden.

Ich kann mich aber des Eindrucks nicht erwehren, daß bei den angeborenen Gelenkcontracturen noch ein anderer Faktor eine wesentliche Rolle spielen muß. Bei den isolierten Gelenkcontracturen können wir uns durch die Herstellung der im Uterus eingenommenen Zwangslage und dadurch bedingte Annäherung. der Ansatzpunkte des betreffenden Muskels durch funktionelle Anpassung das Eintreten der Contractur erklären. Schwerer verständlich wird aber diese Annahme, wenn es sich bei einem Kinde um eine Contractur sowohl im Hüftgelenk wie auch im Kniegelenk handelt. Es sind nur wenige Fälle davon beschrieben.

So berichtet Sch midt ausführlich von einem Fall von gleichzeitiger angeborener Beugecontractur im Hüft- und Kniegelenk und sagt, daß beide Oberschenkel im Hüftgelenk stark flektiert, nach außen rotiert, nahezu bis zur Frontalebene abduziert lagen, und beiderseits die Außenseite des Unterleibs nahe der vorderen Axillarlinie des Körpers berührten. Beide Kniegelenke waren stark gebeugt und einwärts rotiert, so daß die medialen Seiten des Unterschenkels dicht der Beugeseite des Oberschenkels anlagen. Bei einer derartigen Zwangslage können wir uns also die Beugecontractur im Hüftgelenk durch eine funktionelle Anpassung des Ileopsoas auf Grund der vorher für die Verkürzung dieses Muskels typischen Zwangslage erklären.

Eine Erklärung für das Zustandekommen der Beugecontractur im Kniegelenk auf Grund des früher Gesagten läßt sich aber nicht einwandsfrei erbringen. Unwillkürlich sucht man eine Begründung bei der bekanntesten angeborenen muskulären Contractur, dem angeborenen muskulären Schiefhals. Ich will nun nicht alle Theorien, die für die Entstehung des angeborenen muskulären Schiefhalses geltend gemacht sind, wiederholen. Die Entstehung durch ein Geburtstrauma können wir unberücksichtigt lassen, wenn die Geburt spontan und ohne Kunsthilfe erfolgt ist. Für die Ursache derartiger Schiefhälse hat man dann eine abnorme intra-uterine Belastung angenommen, woduŕch die Ursprungs- und Ansatzpunkte des Kopfnickermuskels extrem genähert werden.

Nun hat Voelcker eine große Zahl operativ gewonnener, resezierter Muskelstücke derartiger Schiefhälse untersucht und das pathologisch-anatomische Bild einer Dystrophia fibrosa, d. h. Untergang der 
146 Friedrich Loeffler: Über die angeborenen Contracturen des Hüftgelenks usw.

spezifischen Muskelelemente und Vermehrung der fibrösen Muskelsubstanz gefunden.

Dieses selbe pathologisch-anatomische Bild zeigen nun die ischiämischen Muskeln. Voelcker glaubt daher, daß es sich bei dem intrauterin entstandenen muskulären Schiefhals um eime Ischiämie des Musculus sternocleido-mastoideus handelt, verursacht durch Druck der Schulter gegen diesen Muskel. So gibt er auch gleichzeitig eine glaubwürdige Erkzlärung für den so mannigfachen klinischen Befund bei einem angeborenen muskulären Schiefhals, wenn wir bald den stemalen, bald den clavicularen, bald beide Muskelansätze stark verkürzt finden.

In nenester Zeit hat Brücke durch experimentelle Untersuchungen nachgewiesen, daß die Unterbrechung der Blut- und Lymphzírkulation durch direkten Druck den Muskel wesentlich schwerer schädigt als die Unterbrechung des Blutkreislaufes allein.

So möchte ich zu der Annahme neigen, daß bei dem gleichzeitigen Bestehen von angeborener Beugecontractur im Hüft- und Kniegelenk die angeborene Kniecontractur durch einen infolge der Zwangslage hervorgerufenen Druck der Wade auf die Unterschenkelbeugemuskulatur mit gleichzeitiger Behinderung der Blut- und Lymphzirkulation hervorgerufen ist. Es würde alșo auch hier eine Muskelischämie vorliegen.

Leider sind keine pathologisch-anatomischen Untersuchungen derartig verkürzter. Muskeln bei Gelenkcontracturen vorhanden, wodurch ein Beweis für die Richtigkeit dieser Annahme erbracht werden könnte.

Vielleicht haben wir auch in einer Ischiämie der Quadricepsmuskulatur einen Grund, warum wir die Streckcontracturen des Kniegelenks am häufigsten von allen Gelenkcontracturen beobachten, denn es ist denkbar, daß durch die für ein Zustandekommen einer Kniestreckcontractur nötige Zwangslage ein starker Druck der Bauchwand gegen die Quadricepsmuskulatur ausgeübt und hierdurch schließlich eine Behinderung der normalen Blut- und Lymphzirkulation verursacht wird.

Als Ergebnis meiner Beobachtungen möchte ich mich also dahin äußern, daß für das Zustandekommen isolierter angeborenér Contracturen im Hüft- und Kniegelen $\mathrm{k}$ eine $\mathrm{ty}$ pische $\mathrm{Z}$ wangs lage die hauptsächlichste Ursache sein muß, wodurch es zu einer extremen Annäherung der Ansatzpunkte des betreffenden Muskels mit sekundärer funktioneller Anpassung kommt.

Bei dem gleichzeitigen Bestehen von Gelenkcontracturen im Hüft- und Kniegelenk kommt vielleicht als zweiter Grund noch eine Muskelischiämie in Betracht.

Ob alle diese Annahmen auf Richtigkeit beruhen, kann nur durch einwandsfreie Beobachtung und Mitteilung weiterer Fälle und durch pathologisch-anatomische Untersuchungen resezierter Muskeln festgestellt werden. 